\title{
Using Density Functional Theory to Study Neutral and Ionized Stacked Thymine Dimers
}

\author{
Đura Nakarada, Mihajlo Etinski and Milena Petković \\ Faculty of Physical Chemistry, University of Belgrade \\ Studentski trg 12-16, 11158 Belgrade, Serbia

\section{Supporting Information}

- Table S1: Optimized Cartesian coordinates (in $\AA$ ) of the TD1 dimer at M06-2X/6-311++G(2df,2pd) level.

- Table S2: Optimized Cartesian coordinates (in $\AA$ ) of the TD2 dimer at M06-2X/6-311++G(2df,2pd) level.

- Table S3: Optimized Cartesian coordinates (in $\AA$ ) of the TD3 dimer at M06-2X/6-311++G(2df,2pd) level.

- Table S4: Optimized Cartesian coordinates (in $\AA$ ) of the TD4 dimer at M06-2X/6-311++G(2df,2pd) level.

- Table S5: Optimized Cartesian coordinates (in Å) of the TD5 dimer at M06-2X/6-311++G(2df,2pd) level.

- Table S6: Optimized Cartesian coordinates (in Å) of the TD6 dimer at M06-2X/6-311++G(2df,2pd) level.

- Table S7: Optimized Cartesian coordinates (in $\AA$ ) of the MTD1 dimer at M06-2X/6-311++G(2df,2pd) level.

- Table S8: Optimized Cartesian coordinates (in $\AA$ ) of the MTD2 dimer at M06-2X/6-311++G(2df,2pd) level.

- Table S9: Optimized Cartesian coordinates (in $\AA$ ) of the MTD3 dimer at M06-2X/6-311++G(2df,2pd) level.

- Table S10 Optimized Cartesian coordinates (in A) of the MTD4 dimer at M06-2X/6-311++G(2df,2pd) level.

- Table S11: Optimized Cartesian coordinates (in Å) of the MTD5 dimer at M06-2X/6-311++G(2df,2pd) level.

- Table S12: Optimized Cartesian coordinates (in Å) of the MTD6 dimer at M06-2X/6-311++G(2df,2pd) level.

- Table S13: Optimized Cartesian coordinates (in $\AA$ ) of the TD1 ${ }^{+}$dimer at M06-2X/6-311++G(2df,2pd) level.

- Table S14: Optimized Cartesian coordinates (in $\AA$ ) of the TD2 ${ }^{+}$dimer at M06-2X/6-311++G(2df,2pd) level.

- Table S15: Optimized Cartesian coordinates (in $\AA$ ) of the TD3 ${ }^{+}$dimer at M06-2X/6-311++G(2df,2pd) level.

- Table S16: Optimized Cartesian coordinates (in $\AA$ ) of the TD4 ${ }^{+}$dimer at M06-2X/6-311++G(2df,2pd) level.

- Table S17: Optimized Cartesian coordinates (in $\AA$ ) of the TD5 ${ }^{+}$dimer at M06-2X/6-311++G(2df,2pd) level.

- Table S18: Optimized Cartesian coordinates (in $\AA$ ) of the TD6 ${ }^{+}$dimer at M06-2X/6-311++G(2df,2pd) level.

- Figure S1: Optimized T-shaped structure of the TD5 ${ }^{+}$dimer at M06-2X/6-311++G(2df,2pd) level. 
Table S1: Optimized Cartesian coordinates (in $\AA$ ) of the TD1 dimer at M06-2X/6-311++G(2df,2pd) level.

\begin{tabular}{rrrrrrrr}
\hline \hline 7 & 0.000000 & 0.000000 & 0.000000 & 6 & 0.000000 & 0.000000 & 1.381549 \\
7 & 1.265470 & 0.000000 & 1.924061 & 6 & 2.411080 & -0.107184 & 1.175488 \\
6 & 2.399021 & -0.161465 & -0.164098 & 6 & 1.101645 & -0.150574 & -0.840334 \\
8 & 0.951579 & -0.288416 & -2.035881 & 8 & -1.005792 & -0.018118 & 2.048134 \\
1 & -0.916664 & -0.054840 & -0.428840 & 1 & 3.328068 & -0.157458 & 1.746149 \\
1 & 1.299952 & -0.112068 & 2.925014 & 6 & 3.617189 & -0.305907 & -1.014646 \\
1 & 3.673247 & 0.497631 & -1.748302 & 1 & 3.576526 & -1.242426 & -1.574379 \\
1 & 4.520777 & -0.297226 & -0.408028 & 7 & -0.123613 & -2.965394 & 1.047694 \\
6 & -0.785643 & -2.876987 & -0.161676 & 7 & 0.020563 & -3.153650 & -1.242980 \\
6 & 1.370834 & -3.379818 & -1.143547 & 6 & 2.015966 & -3.410102 & 0.031387 \\
6 & 1.244265 & -3.144648 & 1.245762 & 8 & 1.724654 & -3.050910 & 2.355398 \\
8 & -1.947998 & -2.569310 & -0.264378 & 1 & -0.676924 & -2.714240 & 1.858836 \\
1 & 1.882292 & -3.520318 & -2.085736 & 1 & -0.402471 & -2.989718 & -2.142884 \\
6 & 3.485402 & -3.624621 & 0.182941 & 1 & 3.686456 & -4.462549 & 0.849663 \\
1 & 3.953862 & -3.816473 & -0.780527 & 1 & 3.949817 & -2.744738 & 0.632552 \\
\hline \hline
\end{tabular}

Table S2: Optimized Cartesian coordinates (in $\AA$ ) of the TD2 dimer at M06-2X/6-311++G(2df,2pd) level.

\begin{tabular}{rrrrrrrr}
\hline \hline 7 & 0.000000 & 0.000000 & 0.000000 & 6 & 0.000000 & 0.000000 & 1.377890 \\
7 & 1.261051 & 0.000000 & 1.921872 & 6 & 2.394730 & -0.227708 & 1.171299 \\
6 & 2.377597 & -0.353051 & -0.163147 & 6 & 1.087308 & -0.243760 & -0.845185 \\
8 & 0.919187 & -0.365551 & -2.036127 & 8 & -1.013375 & -0.024766 & 2.040616 \\
1 & -0.910144 & 0.034598 & -0.438339 & 1 & 1.297011 & -0.133303 & 2.922185 \\
1 & 3.306033 & -0.318494 & 1.746481 & 6 & 3.581737 & -0.634645 & -1.001851 \\
1 & 4.475665 & -0.707506 & -0.385582 & 1 & 3.723415 & 0.149963 & -1.744636 \\
1 & 3.458147 & -1.572133 & -1.545466 & 7 & 2.591358 & -3.570633 & 1.268366 \\
6 & 1.650042 & -3.051374 & 2.126710 & 7 & 0.398661 & -2.957928 & 1.566620 \\
6 & 0.148581 & -3.204379 & 0.233438 & 6 & 1.095274 & -3.610196 & -0.623739 \\
6 & 2.445071 & -3.826591 & -0.099208 & 8 & 3.393717 & -4.188326 & -0.755153 \\
8 & 1.901107 & -2.694122 & 3.257252 & 1 & 3.522452 & -3.675795 & 1.647309 \\
1 & -0.871289 & -3.025185 & -0.078595 & 1 & -0.317595 & -2.557728 & 2.156465 \\
6 & 0.873356 & -3.825247 & -2.084936 & 1 & -0.176043 & -3.694011 & -2.339852 \\
1 & 1.195346 & -4.823456 & -2.378770 & 1 & 1.457710 & -3.107625 & -2.661673 \\
\hline \hline
\end{tabular}


Table S3: Optimized Cartesian coordinates (in $\AA$ ) of the TD3 dimer at M06-2X/6-311++G(2df,2pd) level.

\begin{tabular}{rrrrrrrr}
\hline \hline 7 & 0.000000 & 0.000000 & 0.000000 & 6 & 0.000000 & 0.000000 & 1.375070 \\
7 & 1.265061 & 0.000000 & 1.917015 & 6 & 2.414078 & -0.107721 & 1.165337 \\
6 & 2.403139 & -0.181589 & -0.172268 & 6 & 1.107020 & -0.113482 & -0.850570 \\
8 & 0.950526 & -0.168758 & -2.047106 & 8 & -1.007096 & -0.013008 & 2.047817 \\
1 & -0.910178 & 0.012773 & -0.447710 & 1 & 3.332983 & -0.142060 & 1.734288 \\
1 & 1.301979 & 0.012881 & 2.922764 & 6 & 3.617854 & -0.357788 & -1.021897 \\
1 & 3.681571 & 0.434152 & -1.767167 & 1 & 4.520553 & -0.350502 & -0.414004 \\
1 & 3.555238 & -1.307145 & -1.555119 & 7 & -0.020149 & -2.958331 & -1.228113 \\
6 & -1.232298 & -2.605059 & -0.676646 & 7 & -1.282423 & -2.775229 & 0.691036 \\
6 & -0.175916 & -3.067398 & 1.449594 & 6 & 1.028118 & -3.313746 & 0.913002 \\
6 & 1.159161 & -3.250885 & -0.544063 & 8 & 2.197874 & -3.415153 & -1.144176 \\
8 & -2.158454 & -2.169788 & -1.318589 & 1 & 0.074320 & -2.771538 & -2.218915 \\
1 & -0.343812 & -3.071828 & 2.517996 & 1 & -2.112737 & -2.412812 & 1.134549 \\
6 & 2.256733 & -3.623578 & 1.703038 & 1 & 2.050415 & -3.597959 & 2.771646 \\
1 & 2.646768 & -4.607248 & 1.441980 & 1 & 3.044393 & -2.905059 & 1.470534 \\
\hline \hline
\end{tabular}

Table S4: Optimized Cartesian coordinates (in $\AA$ ) of the TD4 dimer at M06-2X/6-311++G(2df,2pd) level.

\begin{tabular}{rrrrrrrr}
\hline \hline 7 & 0.000000 & 0.000000 & 0.000000 & 6 & 0.000000 & 0.000000 & 1.377761 \\
7 & 1.265634 & 0.000000 & 1.925659 & 6 & 2.403241 & -0.190416 & 1.180862 \\
6 & 2.387747 & -0.289584 & -0.156389 & 6 & 1.094940 & -0.204596 & -0.838768 \\
8 & 0.945221 & -0.317654 & -2.034947 & 8 & -1.005372 & -0.018005 & 2.047253 \\
1 & 1.295744 & -0.121033 & 2.926617 & 1 & -0.912621 & -0.078624 & -0.431799 \\
1 & 3.316449 & -0.267953 & 1.755081 & 6 & 3.599937 & -0.507934 & -1.000526 \\
1 & 4.492838 & -0.600857 & -0.384701 & 1 & 3.481435 & -1.410440 & -1.602335 \\
1 & 3.737622 & 0.316162 & -1.700379 & 7 & 2.566258 & -3.594962 & 0.886608 \\
6 & 1.642804 & -3.106758 & 1.782697 & 7 & 0.395125 & -2.930137 & 1.232318 \\
6 & 0.028991 & -3.081093 & -0.111183 & 6 & 1.103500 & -3.533417 & -0.997493 \\
6 & 2.302778 & -3.777883 & -0.452787 & 8 & 1.912736 & -2.847772 & 2.934679 \\
8 & -1.096533 & -2.823944 & -0.466593 & 1 & -0.324851 & -2.583404 & 1.857173 \\
1 & 3.488269 & -3.748403 & 1.259987 & 1 & 3.141034 & -4.122917 & -1.042320 \\
6 & 0.790384 & -3.661480 & -2.451456 & 1 & -0.058449 & -4.327241 & -2.602780 \\
1 & 1.648248 & -4.045021 & -3.000470 & 1 & 0.518176 & -2.684731 & -2.853193 \\
\hline \hline
\end{tabular}


Table S5: Optimized Cartesian coordinates (in $\AA$ ) of the TD5 dimer at M06-2X/6-311++G(2df,2pd) level.

\begin{tabular}{rrrrrrrr}
\hline \hline 7 & 0.000000 & 0.000000 & 0.000000 & 6 & 0.000000 & 0.000000 & 1.379203 \\
7 & 1.266983 & 0.000000 & 1.913877 & 6 & 2.412140 & -0.012962 & 1.149392 \\
6 & 2.400108 & -0.031933 & -0.191686 & 6 & 1.097951 & -0.059194 & -0.860080 \\
8 & 0.928259 & -0.139597 & -2.055991 & 8 & -1.009151 & -0.008155 & 2.042870 \\
1 & -0.913096 & -0.006996 & -0.432860 & 1 & 1.307218 & -0.094045 & 2.916774 \\
1 & 3.336117 & -0.013810 & 1.711377 & 6 & 3.620345 & -0.070524 & -1.051600 \\
1 & 4.522884 & -0.031654 & -0.444837 & 1 & 3.623460 & 0.768091 & -1.747337 \\
1 & 3.624987 & -0.987893 & -1.639454 & 7 & 1.424622 & -3.146688 & 0.962054 \\
6 & 1.424616 & -3.146703 & -0.417148 & 7 & 0.157630 & -3.146710 & -0.951817 \\
6 & -0.987523 & -3.133760 & -0.187327 & 6 & -0.975486 & -3.114776 & 1.153751 \\
6 & 0.326675 & -3.087484 & 1.822138 & 8 & 0.496372 & -3.007068 & 3.018048 \\
8 & 2.433765 & -3.138555 & -1.080819 & 1 & 2.337720 & -3.139686 & 1.394910 \\
1 & -1.911502 & -3.132916 & -0.749307 & 1 & 0.117391 & -3.052676 & -1.954715 \\
6 & -2.195720 & -3.076197 & 2.013670 & 1 & -3.098261 & -3.115088 & 1.406911 \\
1 & -2.198818 & -3.914805 & 2.709415 & 1 & -2.200384 & -2.158815 & 2.601501 \\
\hline \hline
\end{tabular}

Table S6: Optimized Cartesian coordinates (in $\AA$ ) of the TD6 dimer at M06-2X/6-311++G(2df,2pd) level.

\begin{tabular}{rrrrrrrr}
\hline \hline 7 & 0.000000 & 0.000000 & 0.000000 & 6 & 0.000000 & 0.000000 & 1.376633 \\
7 & 1.268737 & 0.000000 & 1.916487 & 6 & 2.419525 & -0.037983 & 1.160518 \\
6 & 2.408164 & -0.074620 & -0.178604 & 6 & 1.105455 & -0.079279 & -0.848551 \\
8 & 0.951416 & -0.169978 & -2.045568 & 8 & -1.002483 & -0.004427 & 2.051119 \\
1 & -0.909501 & -0.083555 & -0.440853 & 1 & 1.306064 & 0.028259 & 2.921695 \\
1 & 3.341300 & -0.041334 & 1.725881 & 6 & 3.629788 & -0.145452 & -1.034978 \\
1 & 4.530989 & -0.093647 & -0.427177 & 1 & 3.636632 & 0.672418 & -1.754688 \\
1 & 3.637542 & -1.079909 & -1.595394 & 7 & 0.565444 & -2.994872 & -0.873783 \\
6 & 1.754936 & -3.346177 & -0.276443 & 7 & 1.613284 & -3.679040 & 1.054072 \\
6 & 0.414330 & -3.626829 & 1.730109 & 6 & -0.731678 & -3.248362 & 1.148605 \\
6 & -0.685436 & -2.872637 & -0.266570 & 8 & -1.631942 & -2.456885 & -0.895920 \\
8 & 2.818925 & -3.359899 & -0.848865 & 1 & 0.633321 & -2.662741 & -1.829610 \\
1 & 2.459605 & -3.968318 & 1.515259 & 1 & 0.461535 & -3.909666 & 2.772747 \\
6 & -2.046281 & -3.150023 & 1.850677 & 1 & -1.961019 & -3.493131 & 2.879888 \\
1 & -2.797224 & -3.748144 & 1.335626 & 1 & -2.390519 & -2.116180 & 1.852647 \\
\hline \hline
\end{tabular}


Table S7: Optimized Cartesian coordinates (in $\AA$ ) of the MTD1 dimer at M06-2X/6-311++G(2df,2pd) level.

\begin{tabular}{rrrrrrrr}
\hline \hline 7 & 0.000000 & 0.000000 & 0.000000 & 6 & 0.000000 & 0.000000 & 1.381549 \\
7 & 0.000000 & 0.000000 & 0.000000 & 6 & 0.000000 & 0.000000 & 1.390026 \\
6 & 1.326267 & 0.000000 & 1.993798 & 6 & 2.390317 & 0.062313 & 1.176136 \\
7 & 2.309242 & 0.104054 & -0.192479 & 6 & 1.090438 & 0.143049 & -0.836418 \\
8 & 0.979958 & 0.305002 & -2.030491 & 8 & -1.044435 & 0.019703 & 2.011122 \\
1 & -0.899848 & 0.080547 & -0.459951 & 1 & 3.398769 & 0.097580 & 1.566908 \\
6 & 3.497381 & 0.317425 & -1.012554 & 6 & 1.410855 & -0.009942 & 3.484515 \\
1 & 4.367124 & 0.002152 & -0.443514 & 1 & 3.413734 & -0.274044 & -1.918812 \\
1 & 3.587564 & 1.371795 & -1.268048 & 1 & 2.447107 & -0.018619 & 3.817523 \\
1 & 0.897878 & -0.879130 & 3.895196 & 1 & 0.916270 & 0.872317 & 3.895974 \\
7 & 0.452657 & 3.034994 & -0.375989 & 6 & -0.889314 & 2.860414 & -0.097129 \\
7 & -1.217211 & 3.011177 & 1.233817 & 6 & -0.249415 & 3.184500 & 2.190244 \\
6 & 1.064452 & 3.278151 & 1.926357 & 6 & 1.491948 & 3.165396 & 0.537810 \\
8 & -1.702032 & 2.582626 & -0.949520 & 8 & 2.649317 & 3.159859 & 0.167059 \\
1 & 0.708505 & 2.873642 & -1.343602 & 1 & -0.624056 & 3.228747 & 3.204447 \\
6 & -2.608721 & 2.772295 & 1.603091 & 6 & 2.122556 & 3.431461 & 2.968663 \\
1 & 1.684442 & 3.515189 & 3.961553 & 1 & 2.732790 & 4.312846 & 2.773560 \\
1 & 2.795295 & 2.571551 & 2.952130 & 1 & -2.772989 & 3.176156 & 2.597864 \\
1 & -3.255905 & 3.272010 & 0.889248 & 1 & -2.814877 & 1.703368 & 1.601551 \\
\hline \hline
\end{tabular}

Table S8: Optimized Cartesian coordinates (in $\AA$ ) of the MTD2 dimer at M06-2X/6-311++G(2df,2pd) level.

\begin{tabular}{rrrrrrrr}
\hline \hline 7 & 0.000000 & 0.000000 & 0.000000 & 6 & 0.000000 & 0.000000 & 1.377087 \\
7 & 1.255330 & 0.000000 & 1.940359 & 6 & 2.389426 & -0.014689 & 1.159107 \\
6 & 2.387918 & -0.033935 & -0.183941 & 6 & 1.095771 & -0.027871 & -0.862132 \\
8 & 0.928856 & -0.051590 & -2.061200 & 8 & -1.021583 & -0.004636 & 2.031404 \\
1 & -0.912051 & -0.000500 & -0.434981 & 1 & 3.316333 & -0.019126 & 1.717358 \\
6 & 1.315109 & -0.020499 & 3.398744 & 6 & 3.616210 & -0.086777 & -1.032437 \\
1 & 4.514383 & -0.067009 & -0.418071 & 1 & 3.639418 & 0.755662 & -1.723265 \\
1 & 3.610065 & -0.999397 & -1.628686 & 1 & 2.357833 & 0.023452 & 3.698400 \\
1 & 0.779690 & 0.836611 & 3.800139 & 1 & 0.859475 & -0.936332 & 3.768384 \\
7 & 1.264345 & -3.118092 & 0.650596 & 6 & 0.209503 & -3.074002 & 1.534753 \\
7 & -1.028730 & -3.074736 & 0.934825 & 6 & -1.158929 & -3.102005 & -0.435959 \\
6 & -0.128919 & -3.125748 & -1.297741 & 6 & 1.220918 & -3.134230 & -0.743220 \\
8 & 2.247016 & -3.146425 & -1.385997 & 8 & 0.364903 & -3.033139 & 2.737236 \\
1 & 2.183697 & -3.117902 & 1.069921 & 1 & -2.182184 & -3.093552 & -0.787665 \\
6 & -0.267599 & -3.118473 & -2.785071 & 6 & -2.183957 & -3.008430 & 1.824733 \\
1 & -2.160686 & -2.074441 & 2.381610 & 1 & -2.160046 & -3.844059 & 2.520133 \\
1 & -3.084280 & -3.058515 & 1.219828 & 1 & 0.234042 & -3.982873 & -3.219414 \\
1 & 0.206709 & -2.225434 & -3.192427 & 1 & -1.315731 & -3.131947 & -3.077966 \\
\hline \hline
\end{tabular}


Table S9: Optimized Cartesian coordinates (in Å) of the MTD3 dimer at M06-2X/6-311++G(2df,2pd) level.

\begin{tabular}{rrrrrrrr}
\hline \hline 7 & 0.000000 & 0.000000 & 0.000000 & 6 & 0.000000 & 0.000000 & 1.375587 \\
7 & 1.254620 & 0.000000 & 1.943414 & 6 & 2.392699 & -0.090371 & 1.170802 \\
6 & 2.391679 & -0.149578 & -0.168516 & 6 & 1.102631 & -0.109435 & -0.852326 \\
8 & 0.945730 & -0.183018 & -2.049337 & 8 & -1.018114 & -0.011977 & 2.037320 \\
1 & -0.910880 & 0.001940 & -0.444956 & 1 & 3.316495 & -0.121717 & 1.733205 \\
6 & 3.617271 & -0.305501 & -1.006141 & 6 & 1.311449 & -0.074038 & 3.395015 \\
1 & 2.343020 & 0.054370 & 3.708816 & 1 & 0.692421 & 0.706318 & 3.828809 \\
1 & 0.941254 & -1.040000 & 3.739969 & 1 & 4.514939 & -0.263579 & -0.392462 \\
1 & 3.664445 & 0.474367 & -1.765204 & 1 & 3.585871 & -1.266962 & -1.519648 \\
7 & 0.115818 & -2.984942 & -1.029831 & 6 & -1.170448 & -2.693937 & -0.629636 \\
7 & -1.389070 & -2.818940 & 0.729336 & 6 & -0.355661 & -3.066085 & 1.594968 \\
6 & 0.917629 & -3.260508 & 1.211324 & 6 & 1.220643 & -3.217703 & -0.216206 \\
8 & 2.326776 & -3.356791 & -0.692861 & 8 & -2.038125 & -2.344854 & -1.397358 \\
1 & 0.304970 & -2.822824 & -2.011531 & 1 & -0.639028 & -3.080392 & 2.639357 \\
6 & -2.731617 & -2.507502 & 1.207162 & 6 & 2.048160 & -3.507604 & 2.155830 \\
1 & 1.695657 & -3.555546 & 3.184958 & 1 & 2.556856 & -4.440076 & 1.912408 \\
1 & 2.795898 & -2.716116 & 2.071721 & 1 & -3.458254 & -3.088464 & 0.646133 \\
1 & -2.939021 & -1.447263 & 1.081765 & 1 & -2.785281 & -2.758185 & 2.262085 \\
\hline \hline
\end{tabular}

Table S10: Optimized Cartesian coordinates (in $\AA$ ) of the MTD4 dimer at M06-2X/6-311++G(2df,2pd) level.

\begin{tabular}{rrrrrrrr}
\hline \hline 7 & 0.000000 & 0.000000 & 0.000000 & 6 & 0.000000 & 0.000000 & 1.375587 \\
7 & 1.254620 & 0.000000 & 1.943415 & 6 & 2.392699 & 0.090412 & 1.170805 \\
6 & 2.391702 & 0.149644 & -0.168513 & 6 & 1.102629 & 0.109474 & -0.852328 \\
8 & 0.945725 & 0.183069 & -2.049337 & 8 & -1.018118 & 0.011959 & 2.037312 \\
1 & -0.910881 & -0.001940 & -0.444956 & 1 & 3.316493 & 0.121753 & 1.733209 \\
6 & 1.311455 & 0.074037 & 3.395016 & 6 & 3.617307 & 0.305611 & -1.006112 \\
1 & 3.664513 & -0.474242 & -1.765189 & 1 & 3.585902 & 1.267087 & -1.519589 \\
1 & 4.514965 & 0.263681 & -0.392420 & 1 & 2.343032 & -0.054334 & 3.708811 \\
1 & 0.692472 & -0.706353 & 3.828812 & 1 & 0.941210 & 1.039977 & 3.739975 \\
7 & 0.115860 & 2.984985 & -1.029816 & 6 & -1.170400 & 2.693948 & -0.629627 \\
7 & -1.389020 & 2.818903 & 0.729348 & 6 & -0.355612 & 3.066035 & 1.594986 \\
6 & 0.917676 & 3.260490 & 1.211344 & 6 & 1.220683 & 3.217747 & -0.216207 \\
8 & 2.326802 & 3.356899 & -0.692874 & 8 & -2.038079 & 2.344895 & -1.397360 \\
1 & 0.305012 & 2.822902 & -2.011521 & 1 & -0.638975 & 3.080304 & 2.639376 \\
6 & 2.048206 & 3.507579 & 2.155853 & 6 & -2.731568 & 2.507451 & 1.207166 \\
1 & 2.556882 & 4.440066 & 1.912457 & 1 & 2.795960 & 2.716107 & 2.071717 \\
1 & 1.695707 & 3.555484 & 3.184984 & 1 & -2.785230 & 2.758096 & 2.262097 \\
1 & -3.458205 & 3.088435 & 0.646158 & 1 & -2.938975 & 1.447217 & 1.081732 \\
\hline \hline
\end{tabular}


Table S11: Optimized Cartesian coordinates (in $\AA$ ) of the MTD5 dimer at M06-2X/6-311++G(2df,2pd) level.

\begin{tabular}{rrrrrrrr}
\hline \hline 7 & 0.000000 & 0.000000 & 0.000000 & 6 & 0.000000 & 0.000000 & 1.379723 \\
7 & 1.257923 & 0.000000 & 1.940602 & 6 & 2.389084 & -0.003158 & 1.158373 \\
6 & 2.386125 & 0.007903 & -0.185628 & 6 & 1.093336 & 0.021126 & -0.860369 \\
8 & 0.925897 & 0.056272 & -2.061790 & 8 & -1.018813 & 0.003797 & 2.033333 \\
1 & -0.915191 & 0.005463 & -0.428325 & 1 & 3.317414 & -0.006817 & 1.714437 \\
6 & 1.317497 & 0.035928 & 3.398735 & 6 & 3.615056 & 0.035918 & -1.034763 \\
1 & 3.625391 & -0.811332 & -1.720051 & 1 & 3.625196 & 0.945210 & -1.635659 \\
1 & 4.512467 & 0.004976 & -0.419719 & 1 & 2.360726 & 0.011978 & 3.698952 \\
1 & 0.794529 & -0.824431 & 3.809716 & 1 & 0.846984 & 0.948742 & 3.757371 \\
7 & 1.304055 & 3.110083 & 0.666314 & 6 & 1.304049 & 3.110089 & -0.713409 \\
7 & 0.046123 & 3.110103 & -1.274282 & 6 & -1.085034 & 3.113268 & -0.492049 \\
6 & -1.082068 & 3.102201 & 0.851952 & 6 & 0.210722 & 3.088963 & 1.526687 \\
8 & 0.378166 & 3.053811 & 2.728107 & 8 & 2.322858 & 3.106286 & -1.367023 \\
1 & 2.219246 & 3.104610 & 1.094634 & 1 & -2.013366 & 3.116938 & -1.048109 \\
6 & -0.013485 & 3.074172 & -2.732414 & 6 & -2.310997 & 3.074171 & 1.701092 \\
1 & -3.208410 & 3.105109 & 1.086054 & 1 & -2.321336 & 3.921418 & 2.386384 \\
1 & -2.321127 & 2.164877 & 2.301985 & 1 & -1.056720 & 3.098133 & -3.032607 \\
1 & 0.457026 & 2.161360 & -3.091059 & 1 & 0.509468 & 3.934533 & -3.143408 \\
\hline \hline
\end{tabular}

Table S12: Optimized Cartesian coordinates (in $\AA$ ) of the MTD6 dimer at M06-2X/6-311++G(2df,2pd) level.

\begin{tabular}{rrrrrrrr}
\hline \hline 7 & 0.000000 & 0.000000 & 0.000000 & 6 & 0.000000 & 0.000000 & 1.376554 \\
7 & 1.260269 & 0.000000 & 1.942571 & 6 & 2.398457 & 0.024624 & 1.167246 \\
6 & 2.396852 & 0.015263 & -0.174227 & 6 & 1.102913 & -0.024544 & -0.853706 \\
8 & 0.951762 & -0.091926 & -2.052404 & 8 & -1.010257 & -0.007777 & 2.043107 \\
1 & -0.912207 & -0.065164 & -0.437660 & 1 & 3.324400 & 0.049559 & 1.727187 \\
6 & 1.314541 & -0.012699 & 3.396284 & 6 & 3.628438 & 0.014599 & -1.019481 \\
1 & 4.522857 & 0.063301 & -0.400627 & 1 & 3.662550 & -0.893174 & -1.621167 \\
1 & 3.622202 & 0.863753 & -1.702330 & 1 & 2.354860 & -0.059655 & 3.704557 \\
1 & 0.849164 & 0.883220 & 3.802192 & 1 & 0.773760 & -0.876234 & 3.779823 \\
7 & 0.463065 & -2.973450 & -1.015776 & 6 & 1.700067 & -3.141876 & -0.436276 \\
7 & 1.666983 & -3.306503 & 0.934734 & 6 & 0.479341 & -3.305877 & 1.633011 \\
6 & -0.723052 & -3.119395 & 1.069436 & 6 & -0.767548 & -2.872135 & -0.370861 \\
8 & -1.765968 & -2.577685 & -0.989679 & 8 & 2.735629 & -3.138336 & -1.063729 \\
1 & 0.477520 & -2.731599 & -1.999484 & 1 & 0.581897 & -3.465398 & 2.698599 \\
6 & 2.952804 & -3.456373 & 1.596005 & 6 & -2.011090 & -3.082483 & 1.824375 \\
1 & -2.711742 & -3.811955 & 1.419030 & 1 & -2.467434 & -2.097529 & 1.733189 \\
1 & -1.846964 & -3.292739 & 2.879646 & 1 & 2.787496 & -3.517863 & 2.667902 \\
1 & 3.457850 & -4.355254 & 1.248078 & 1 & 3.587060 & -2.601550 & 1.364448 \\
\hline \hline
\end{tabular}


Table S13: Optimized Cartesian coordinates (in $\AA$ ) of the TD1 ${ }^{+}$dimer at M06-2X/6-311++G(2df,2pd) level.

\begin{tabular}{rrrrrrrr}
\hline \hline 7 & -0.020384 & 0.013227 & -0.039515 & 6 & -0.004977 & 0.069572 & 1.336699 \\
7 & 1.289402 & -0.056805 & 1.883316 & 6 & 2.394096 & -0.259322 & 1.161758 \\
6 & 2.361225 & -0.382806 & -0.210435 & 6 & 1.056708 & -0.241133 & -0.882913 \\
8 & 0.901220 & -0.378828 & -2.070155 & 8 & -0.967770 & 0.184732 & 2.031435 \\
1 & -0.933183 & 0.115559 & -0.472213 & 1 & 3.318999 & -0.345968 & 1.717742 \\
1 & 1.322632 & -0.047934 & 2.896689 & 6 & 3.587725 & -0.444532 & -1.047909 \\
1 & 3.802304 & 0.547837 & -1.453562 & 1 & 3.439140 & -1.097905 & -1.905876 \\
1 & 4.450656 & -0.770081 & -0.471232 & 7 & -0.124996 & -2.975440 & 1.093366 \\
6 & -0.785706 & -2.945574 & -0.114916 & 7 & 0.073636 & -3.107007 & -1.222011 \\
6 & 1.399956 & -3.229368 & -1.133093 & 6 & 2.060556 & -3.189782 & 0.075744 \\
6 & 1.249254 & -3.048694 & 1.298836 & 8 & 1.721398 & -2.953322 & 2.403671 \\
8 & -1.958135 & -2.775410 & -0.253993 & 1 & -0.711616 & -2.877381 & 1.916266 \\
1 & 1.933801 & -3.337661 & -2.068696 & 1 & -0.386410 & -3.058821 & -2.124311 \\
6 & 3.510385 & -3.485735 & 0.216742 & 1 & 3.641277 & -4.524284 & 0.532460 \\
1 & 4.040932 & -3.346387 & -0.722561 & 1 & 3.957434 & -2.872275 & 0.997059 \\
\hline \hline
\end{tabular}

Table S14: Optimized Cartesian coordinates (in $\AA$ ) of the TD2 ${ }^{+}$dimer at M06-2X/6-311++G(2df,2pd) level.

\begin{tabular}{rrrrrrrr}
\hline \hline 7 & -0.056442 & -0.007057 & -0.139957 & 6 & -0.025475 & -0.057132 & 1.230299 \\
7 & 1.271712 & -0.261148 & 1.748641 & 6 & 2.360475 & -0.482584 & 0.995092 \\
6 & 2.299526 & -0.545558 & -0.375135 & 6 & 1.001803 & -0.264572 & -1.019944 \\
8 & 0.836112 & -0.270423 & -2.209007 & 8 & -0.976151 & 0.024844 & 1.951097 \\
1 & -0.956269 & 0.202769 & -0.558173 & 1 & 1.331227 & -0.240659 & 2.761590 \\
1 & 3.292407 & -0.616933 & 1.529741 & 6 & 3.484722 & -0.759140 & -1.234701 \\
1 & 4.399166 & -0.819542 & -0.650591 & 1 & 3.559591 & 0.046336 & -1.967620 \\
1 & 3.387668 & -1.685315 & -1.811351 & 7 & 2.610364 & -3.596861 & 1.494908 \\
6 & 1.609485 & -3.247079 & 2.364942 & 7 & 0.362982 & -3.058722 & 1.729524 \\
6 & 0.175445 & -3.115784 & 0.401428 & 6 & 1.207825 & -3.353003 & -0.472146 \\
6 & 2.538150 & -3.644064 & 0.097363 & 8 & 3.507057 & -3.886436 & -0.568843 \\
8 & 1.738800 & -3.075257 & 3.541296 & 1 & 3.510603 & -3.806817 & 1.912172 \\
1 & -0.837810 & -2.956305 & 0.054617 & 1 & -0.407665 & -2.859466 & 2.359150 \\
6 & 1.029251 & -3.450494 & -1.937671 & 1 & -0.014601 & -3.350164 & -2.222888 \\
1 & 1.426820 & -4.401918 & -2.295858 & 1 & 1.599332 & -2.670469 & -2.453730 \\
\hline \hline
\end{tabular}


Table S15: Optimized Cartesian coordinates (in $\AA$ ) of the TD3 ${ }^{+}$dimer at M06-2X/6-311++G(2df,2pd) level.

\begin{tabular}{rrrrrrrr}
\hline \hline 7 & 0.511449 & -0.326826 & 0.218940 & 6 & 0.831867 & -0.470687 & 1.543972 \\
7 & 2.129626 & -0.165404 & 1.823917 & 6 & 3.051818 & 0.198870 & 0.854579 \\
6 & 2.764153 & 0.214898 & -0.454095 & 6 & 1.404358 & -0.141455 & -0.853916 \\
8 & 1.007077 & -0.298635 & -1.982004 & 8 & 0.034041 & -0.891942 & 2.369174 \\
1 & -0.466280 & -0.412664 & -0.023108 & 1 & 4.034254 & 0.443282 & 1.232222 \\
1 & 2.391817 & -0.196037 & 2.797624 & 6 & 3.742096 & 0.537087 & -1.535025 \\
1 & 3.377036 & 1.363224 & -2.143922 & 1 & 4.710487 & 0.802514 & -1.118815 \\
1 & 3.862175 & -0.320110 & -2.197036 & 7 & -0.043892 & -3.068281 & -1.158733 \\
6 & -1.384194 & -2.921157 & -0.931245 & 7 & -1.749746 & -3.048075 & 0.456754 \\
6 & -0.914381 & -3.264480 & 1.446534 & 6 & 0.456536 & -3.440130 & 1.195489 \\
6 & 0.943031 & -3.243809 & -0.196185 & 8 & 2.112150 & -3.210375 & -0.459708 \\
8 & -2.226170 & -2.687328 & -1.734674 & 1 & 0.272063 & -2.839355 & -2.098930 \\
1 & -1.314279 & -3.305762 & 2.451309 & 1 & -2.744289 & -2.921150 & 0.625287 \\
6 & 1.434587 & -3.741257 & 2.247166 & 1 & 0.994799 & -3.705800 & 3.238845 \\
1 & 1.873707 & -4.728225 & 2.064393 & 1 & 2.268828 & -3.038790 & 2.170513 \\
\hline \hline
\end{tabular}

Table S16: Optimized Cartesian coordinates (in $\AA$ ) of the TD4 ${ }^{+}$dimer at M06-2X/6-311++G(2df,2pd) level.

\begin{tabular}{rrrrrrrr}
\hline \hline 7 & 0.081874 & 0.101751 & 0.020436 & 6 & -0.205222 & 0.378490 & 1.328410 \\
7 & 0.960587 & 0.439814 & 2.173044 & 6 & 2.196864 & 0.238570 & 1.778776 \\
6 & 2.480036 & -0.018604 & 0.427123 & 6 & 1.335130 & -0.184361 & -0.507592 \\
8 & 1.486792 & -0.568628 & -1.633088 & 8 & -1.278947 & 0.541698 & 1.807552 \\
1 & 0.742949 & 0.628597 & 3.148044 & 1 & -0.719584 & -0.096045 & -0.575169 \\
1 & 2.982213 & 0.280425 & 2.522194 & 6 & 3.841965 & -0.195809 & -0.089629 \\
1 & 4.582982 & -0.210822 & 0.703319 & 1 & 3.884683 & -1.118730 & -0.674167 \\
1 & 4.068241 & 0.605070 & -0.802181 & 7 & 2.817408 & -3.714436 & 0.046336 \\
6 & 2.195453 & -2.963647 & 0.997773 & 7 & 0.848523 & -2.821915 & 0.786200 \\
6 & 0.134282 & -3.152872 & -0.381274 & 6 & 0.873642 & -3.987332 & -1.326214 \\
6 & 2.163175 & -4.235633 & -1.059594 & 8 & 2.775331 & -2.411821 & 1.921986 \\
8 & -0.986291 & -2.729568 & -0.523583 & 1 & 0.316302 & -2.387047 & 1.527511 \\
1 & 3.797900 & -3.897629 & 0.197548 & 1 & 2.781110 & -4.837724 & -1.710113 \\
6 & 0.147512 & -4.473298 & -2.536651 & 1 & -0.732334 & -5.046631 & -2.247364 \\
1 & 0.790490 & -5.095342 & -3.153987 & 1 & -0.201784 & -3.626619 & -3.127067 \\
\hline \hline
\end{tabular}


Table S17: Optimized Cartesian coordinates (in $\AA$ ) of the TD5 ${ }^{+}$dimer at M06-2X/6-311++G(2df,2pd) level.

\begin{tabular}{rrrrrrrr}
\hline \hline 7 & 1.099890 & 1.775719 & 0.055587 & 6 & 1.851888 & 2.156017 & 1.136986 \\
7 & 3.032659 & 1.366310 & 1.316574 & 6 & 3.371760 & 0.334519 & 0.575382 \\
6 & 2.552390 & -0.094465 & -0.473441 & 6 & 1.289292 & 0.648266 & -0.721533 \\
8 & 0.486073 & 0.288541 & -1.540827 & 8 & 1.619940 & 3.054357 & 1.877824 \\
1 & 0.241402 & 2.296796 & -0.088662 & 1 & 3.602592 & 1.663506 & 2.103194 \\
1 & 4.292365 & -0.179308 & 0.820745 & 6 & 2.865306 & -1.244980 & -1.332432 \\
1 & 3.806121 & -1.716736 & -1.065851 & 1 & 2.885502 & -0.928571 & -2.378828 \\
1 & 2.051545 & -1.973340 & -1.262577 & 7 & 0.301004 & -2.967405 & 2.118831 \\
6 & 0.337156 & -1.965440 & 1.206258 & 7 & -0.490456 & -2.134406 & 0.141478 \\
6 & -1.326961 & -3.231544 & 0.001846 & 6 & -1.376413 & -4.220590 & 0.902711 \\
6 & -0.507716 & -4.126274 & 2.080569 & 8 & -0.440589 & -4.931017 & 2.967486 \\
8 & 1.072426 & -0.978328 & 1.330689 & 1 & 0.894894 & -2.867253 & 2.932017 \\
1 & -1.938067 & -3.225673 & -0.889204 & 1 & -0.505137 & -1.392386 & -0.545614 \\
6 & -2.259769 & -5.418229 & 0.799589 & 1 & -2.859126 & -5.387789 & -0.106744 \\
1 & -1.660854 & -6.328454 & 0.804028 & 1 & -2.919814 & -5.472442 & 1.664829 \\
\hline \hline
\end{tabular}

Table S18: Optimized Cartesian coordinates (in $\AA$ ) of the TD6 ${ }^{+}$dimer at M06-2X/6-311++G(2df,2pd) level.

\begin{tabular}{rrrrrrrr}
\hline \hline 7 & 0.004722 & -0.201781 & 0.082756 & 6 & -0.067007 & -0.060906 & 1.451995 \\
7 & 1.174884 & -0.099302 & 2.069523 & 6 & 2.362994 & -0.120190 & 1.382717 \\
6 & 2.427460 & -0.235726 & 0.039815 & 6 & 1.162390 & -0.293855 & -0.706053 \\
8 & 1.075507 & -0.450587 & -1.896570 & 8 & -1.098253 & 0.035060 & 2.065865 \\
1 & -0.877217 & -0.135510 & -0.415411 & 1 & 1.158228 & 0.099984 & 3.060021 \\
1 & 3.252563 & -0.040371 & 1.993444 & 6 & 3.691128 & -0.265306 & -0.748502 \\
1 & 4.562659 & -0.198692 & -0.101983 & 1 & 3.706625 & 0.563248 & -1.457105 \\
1 & 3.746556 & -1.180337 & -1.341114 & 7 & 0.340508 & -3.241662 & -1.167126 \\
6 & 1.608060 & -3.311269 & -0.665202 & 7 & 1.652407 & -3.295887 & 0.768399 \\
6 & 0.596942 & -3.232029 & 1.565633 & 6 & -0.688974 & -3.128425 & 1.040345 \\
6 & -0.835762 & -3.075271 & -0.441917 & 8 & -1.892209 & -2.887563 & -0.972176 \\
8 & 2.622912 & -3.379373 & -1.282650 & 1 & 0.272215 & -3.142207 & -2.176388 \\
1 & 2.592964 & -3.384085 & 1.140761 & 1 & 0.773152 & -3.269097 & 2.633073 \\
6 & -1.891088 & -3.005516 & 1.867044 & 1 & -1.718883 & -3.308118 & 2.896403 \\
1 & -2.720698 & -3.553105 & 1.419269 & 1 & -2.193877 & -1.944533 & 1.870089 \\
\hline \hline
\end{tabular}




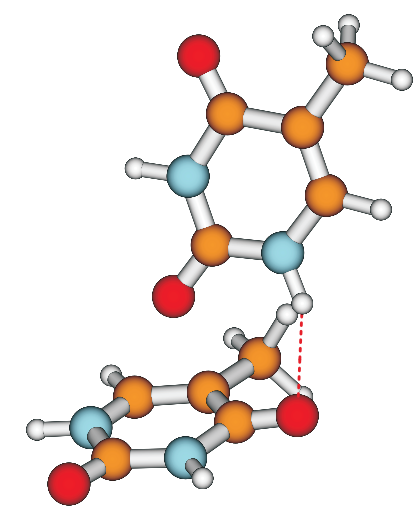

Figure S1: Optimized T-shaped structure of the $\mathrm{TD}^{+}$dimer. 